\section{TAILOR-MADE AFTERCARE PACKS}

Curaprox offer dental practices a new range of tailor-made patient aftercare packs designed to complement the four most common dental treatment scenarios.

The Curaprox Orthodontic Pack consists of a handy white PVC bag containing:

- An orthodontic toothbrush

- A soft $6 \mathrm{~mm}$ Sensitive Sulcular Brush designed to promote precise and efficient cleaning in and around orthodontic braces

- A 75 ml tube of Enzycal

Remineralising Toothpaste, perfect for strengthening and supporting the body's natural immune system against harmful microbes and preventing the formation of mouth ulcers

- A pocket-size CPS Handy Inter-dental brush in a transparent carry case together with a set of three, attachable

\section{SUPERHERO INFECTION CONTROL}

Schülke's Mike Mikrozid will help your practice stay up to date with the best tips on how to adhere to current infection control procedures. Schülke's mikrozid

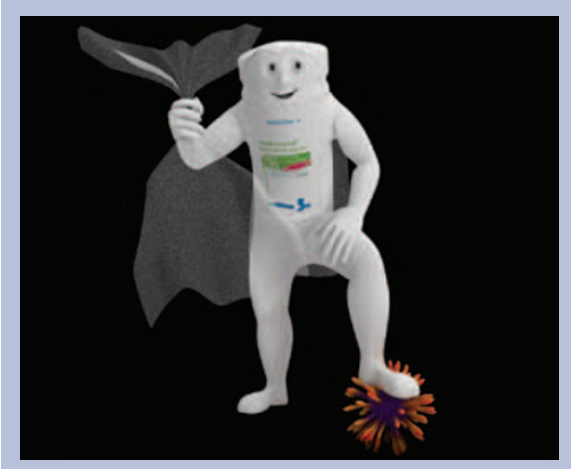

brush heads, colour-coded according to size

- A 'Clean Teeth, Healthy Gums' patient information leaflet.

A Perio Pack and Implant Pack are also available as well as an Oral Medicine Pack for patients suffering from the symptoms of dry mouth.

Reader response number 62

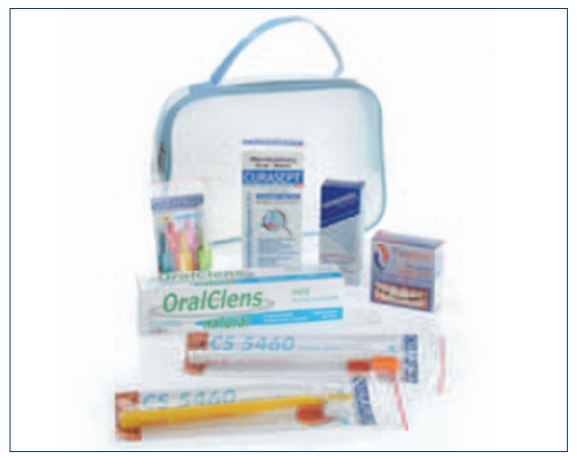

range is kind to delicate surfaces but tough on infection, meaning it is ideal for use throughout the dental surgery.

The mikrozid range of products includes mikrozid sensitive, mikrozid $\mathrm{AF}$ and terralin protect; all of which are well known for their effectiveness against pathogenic bacteria and microorganisms.

Mike Mikrozid recommends that dental professionals look for the best CE marked products available on the market, like those in the mikrozid range. On the mikrozid website Mike will guide you through fun activities to improve your knowledge of infection control. Reader response number 63

\section{A GENTLE INTRODUCTION TO SONIC BRUSHING}

Sonicare has added a new, lower priced power toothbrush to their portfolio making the switch from a manual to a power toothbrush that much easier.

EasyClean offers all the benefits of sonic cleaning technology, combining high speed bristle motion and dynamic fluid action. It has been designed with a simple on/off button and easy start mechanism to gently introduce the user to the sensation of sonic brushing. It also provides failsafe timed brushing thanks to an on-board 'smart timer' which automatically measures brushing in four equally phased periods to ensure consistent and thorough cleaning for two minutes.

The EasyClean brush includes the innovative ProResults brush head with its dynamic bristle motion designed to push fluid into interproximal spaces - this combination of sonic technology and the unique brush head action has been shown to remove more plaque than a manual toothbrush - especially interproximally.

Reader response number 64

\section{A FEW DROPS FIGHT PLAQUE}

Tandex Gel works alongside your patients' daily routine to promote good dental health.

With its sodium fluoride base and added chlorhexidine, clinical studies have shown that using Tandex Gel as part of a daily oral health regimen can help reduce the presence of Streptococcus mutans in the mouth and help protect tooth enamel.

Easy to use and with a pleasant taste, a few drops of Tandex Gel on an interdental brush every day can help maintain good oral hygiene and help keep up the fight against plaque. Reader response number 65

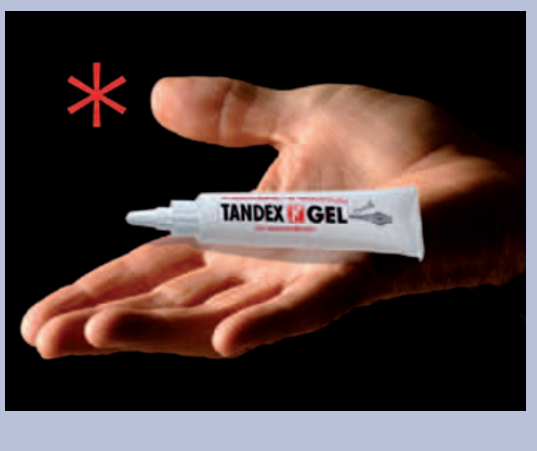

\section{MOUTHWASH TARGETS BAD BREATH}

CB12 is a unique anti-halitosis mouthwash proven to neutralise and prevent production of the foul smelling volatile sulphur compounds (VCSs) which cause endogenous bad breath. When tested against four other commercially available mouthwashes the product came out top in clinical comparison studies carried out by the Microbiology Unit of West of England University, Bristol.

According to its developers, CB12 works by chemically binding itself to tissues in the oral cavity and effectively stopping VSCs in their tracks. The product can be used by anyone over 8-years-old and a single dose remains effective in keeping the breath fresh for 12 hours. Dental practices can order CB12 in quantities as small as one box at a time. Reader response number 66 